\title{
Fabrication and Properties of Amorphous IGZO-TFT
}

\author{
Lu Qingru ${ }^{1,2, \text { a, }{ }^{*} \text {, Huang Xiaodong }}{ }^{3, \text { b }}$, Li Fan ${ }^{3, ~ c}$, Xin Haiyan ${ }^{1}$, Huang Hui ${ }^{1}$ \\ ${ }^{1}$ Department of Electronics, Chengxian Institute, Southeast University, Nanjing, Jiangsu, China \\ ${ }^{2}$ State Key Laboratory of Millimeter Waves, Southeast University, Nanjing, Jiangsu, China \\ ${ }^{3}$ Key Laboratory of MEMS of the Ministry of Education, Southeast University, Nanjing Jiangsu, China \\ a121919084@qq.com, b352812402@qq.com, 'c2863864200@qq.com \\ *Lu Qingru
}

Keywords: IGZO; TFT; Magnetron Sputtering; Active Layer

\begin{abstract}
With the introduction and development of amorphous InGaZnO (a-IGZO or IGZA) in display and other fields, the fabrication and properties of novel semiconductor devices, amorphous IGZO-TFT has become a hot topic in microelectronics and other related disciplines. In this paper, the length and width of IGZO film in IGZO-TFT which is prepared by magnetron sputtering are all $50 \mathrm{um}$. The results show that the threshold voltage of the device is $4.5 \mathrm{~V}$, the sub-threshold swing is small, the gate bias has a good control effect on the leakage current of the device, and the clamping characteristics of the device are also good.
\end{abstract}

\section{Introduction}

The electronics industry has risen to become a major industry in the world since twenty-first Century. High integration and miniaturization of electronic products have been developed with the progress of LSI, especially with the development of display technology. The development of new materials and the technology of making thin film have become the key to realize the miniaturization and integration of the system.

As a new type of transparent oxide semiconductor material, IGZO thin film has good transmittance in visible light band, good compatibility with flexible substrate, uniformity and stable chemical properties, so it is suitable as active layer materials, it also has great application prospects in display industry. Compared with the traditional thin film transistor active layer material (hydrogenated amorphous silicon), IGZO material has more advantages, such as high carrier mobility, low temperature process, good optical transmittance and so on. It can meet the needs of large area liquid crystal display technology, thus has the potential to replace the traditional siliconbased materials. In addition, IGZO is also considered as an important material in the development of flexible display technology and more extensive flexible wearable electronics technology. Therefore, it is of great significance to study the IGZO semiconductor materials.

\section{Structure and fabrication}

As a voltage-controlled device, IGZO-TFT has three external contact poles just like other TFT: gate $\mathrm{G}$, source $\mathrm{S}$ and drain $\mathrm{D}$.

There are three kinds of IGZO transistors in terms of structure classification: bottom-gate, topgate and double-gate. Compared with the latter two structures, the bottom-gate structure has higher effective mobility, larger source-drain current $\left({ }^{I_{s d}}\right)$ and better threshold voltage stability. At the same time, as a display controller device, the bottom-gate structure can ensure that the IGZO film is not affected by substrate illumination, and it has better stability in display applications.

IGZO-TFT with the structure of bottom-gate is fabricated and studied here, the specific structure is as shown in Figure 1. 


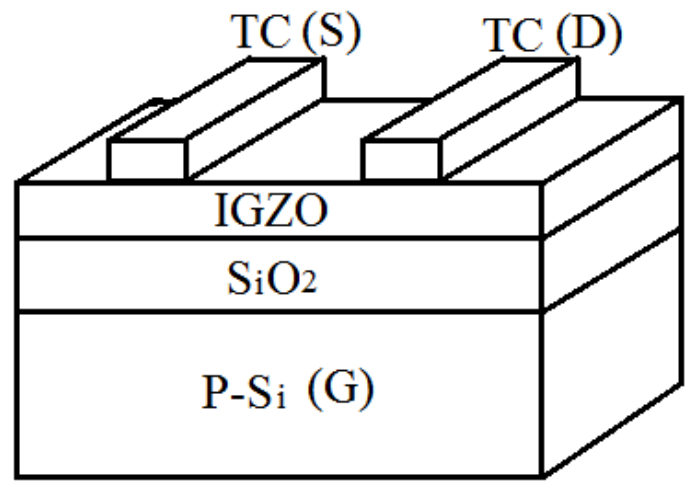

Fig. 1 Bottom-gate structure IGZO-TFT

It is necessary for a P-type silicon wafer heavily doped as the substrate of the TFT to be cleaned and dried to remove the dust, organic matter, metal ions and so on. The cleaned Si sheet was put into the oxidation diffusion furnace, and a layer of $\mathrm{SiO} 2$ film was grown on the surface of Si sheet at $900^{\circ} \mathrm{C}$ in the atmosphere of high purity $\mathrm{O} 2$ through the thermal oxidation process then.

Considering the low density requirement of TFT charge storage layer, it is easier to capture electrons when defects exist. IGZO thin films considered as the charge storage layer are fabricated by magnetron sputtering as the result. Magnetron sputtering, as shown in Fig. 2 (a), is a process in which particles (ions or neutral atoms or molecules) with a certain energy bombard the target surface, so that the atoms or molecules on the target surface can obtain enough energy and eventually escape from the target surface. It can be used to deposit thin films.

The Vacuum Discovery Deposition System (DVDDS) magnetron reactive sputtering system manufactured by Denton Company was used in this experiment, shown in Figure 2 (b). Firstly, the substrate material was put into the sputtering chamber, then the vacuum was pumped by mechanical pump and molecular pump, and the final vacuum was $1.3 \times 10^{-3} \mathrm{~Pa}$. The gases mixed by 45 scem Ar and 5 scem $\mathrm{O} 2$ were filled in and they were under the power of $100 \mathrm{~W}$ RF. The sputtering process was completed according to the set process parameters then, and a layer of IGZO thin film with the length and the width were all $50 \mathrm{um}$ was formed on the surface of SiO2. Titanium alloy (TC: Ti $20 \mathrm{~nm}$, Au $80 \mathrm{~nm}$ ) was evaporated by electron beam, source S and drain D were formed on the IGZO film then with the help of the mask plate, and IGZO-TFT was finally fabricated.

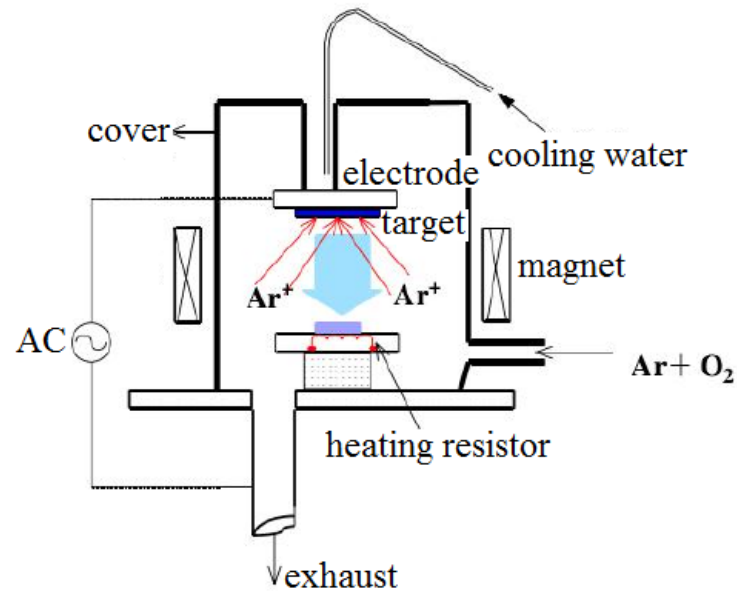

Fig. 2 (a) schematic diagram of magnetron sputtering

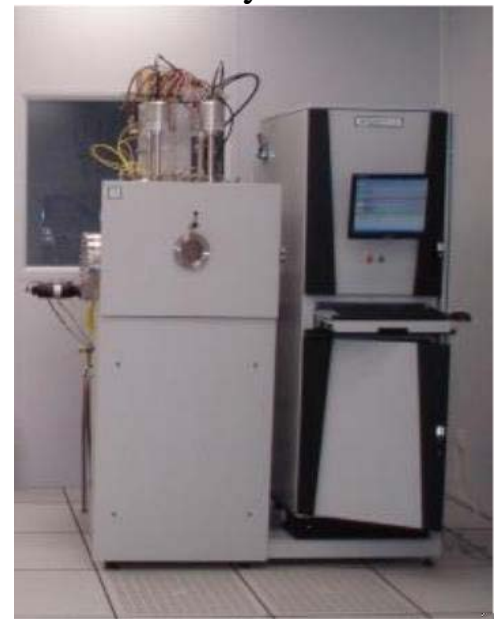

(b) Denton sputtering equipment

\section{Tests and results}

The performance of the new fabricated IGZO-TFT is studied then with the help of the probe and Keithley 4200 Semiconductor Parameter Analyzer. In order to do tests expediently, the conductive silver paste for IGZO-TFT is adhered to the stainless steel blade and dried by the baking table, thus leading to the gate. The probe can be directly tied to the stainless steel during the tests. 
The transfer characteristic curve of IGZO-TFT is shown in Figure 3., the leakage current $I_{d s}$ is very small when the gate voltage $V_{g s}$ is less than $4.5 \mathrm{~V}$, that means the device is in a closed state; $I_{d s}$ increases rapidly when $V_{g s}>4.5 \mathrm{~V}$. It shows that the threshold voltage $V_{t h}$ of the device is $4.5 \mathrm{~V}$. On the other hand, $I_{d s}$ increases exponentially when $V_{g s}$ increases, indicating that the swing of subthreshold is small and the device has good performance in transfer characteristic.

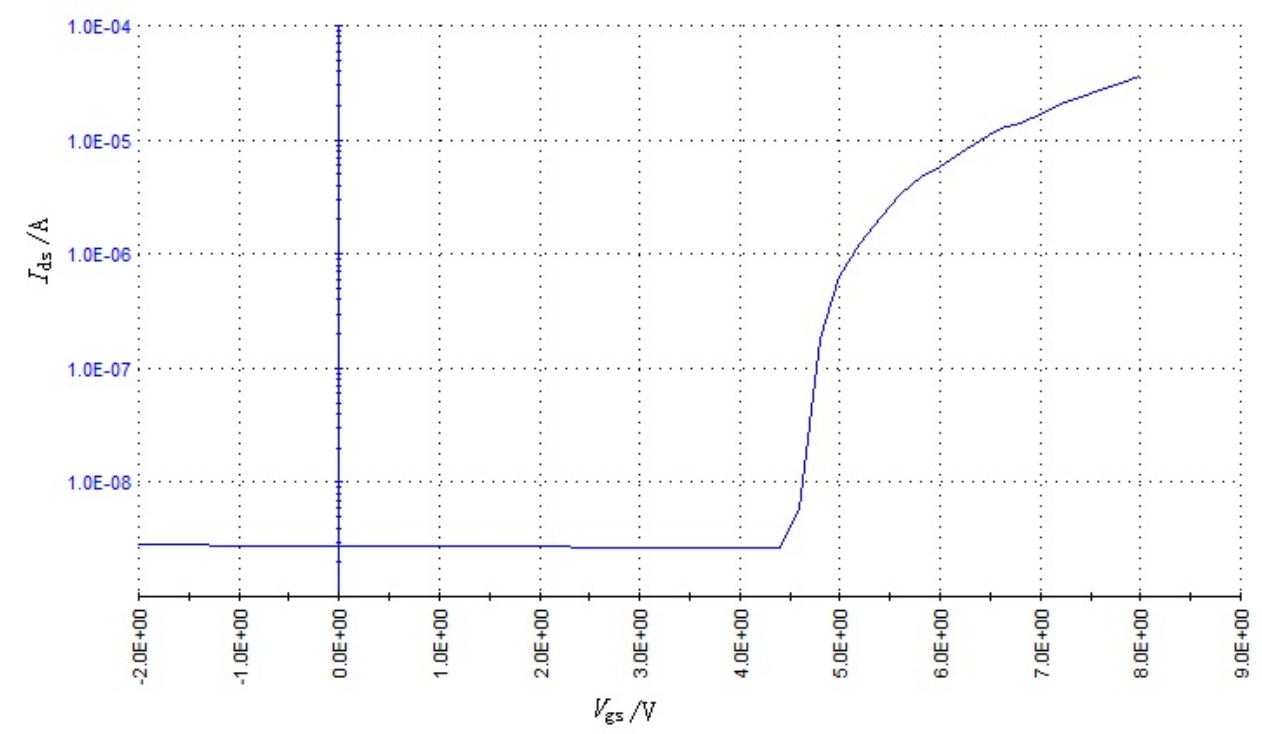

Fig. 3 Transfer characteristic curve

The source $\mathrm{S}$ is grounded and the gate voltages are set to $3 \mathrm{~V}, 4 \mathrm{~V}, 5 \mathrm{~V}, 6 \mathrm{~V}$ respectively. The leakage voltage increases gradually from $0 \mathrm{~V}$ to $6 \mathrm{~V}$ under different gate voltages. The transmission characteristics of the device under different gate voltages are detected. The results are shown in Figure 4. when $V_{g s}<4.5 \mathrm{~V}$, it is almost zero for $I_{d s}$; The channel of the device is turn-on and $I_{d s}$ is no longer zero when $V_{g s}>V_{t h}, I_{d s}$ increases significantly with the increase of $V_{g s}$, but it tends to be a constant then. It shows that $V_{g s}$ has a good regulation effect on $I_{d s}$ for the IGZO-TFT device, and the IGZO-TFT has a good clamping characteristics, there is no obvious rise or fall in the leakage current in the saturation region, which shows that the TFT has good electrical characteristics.

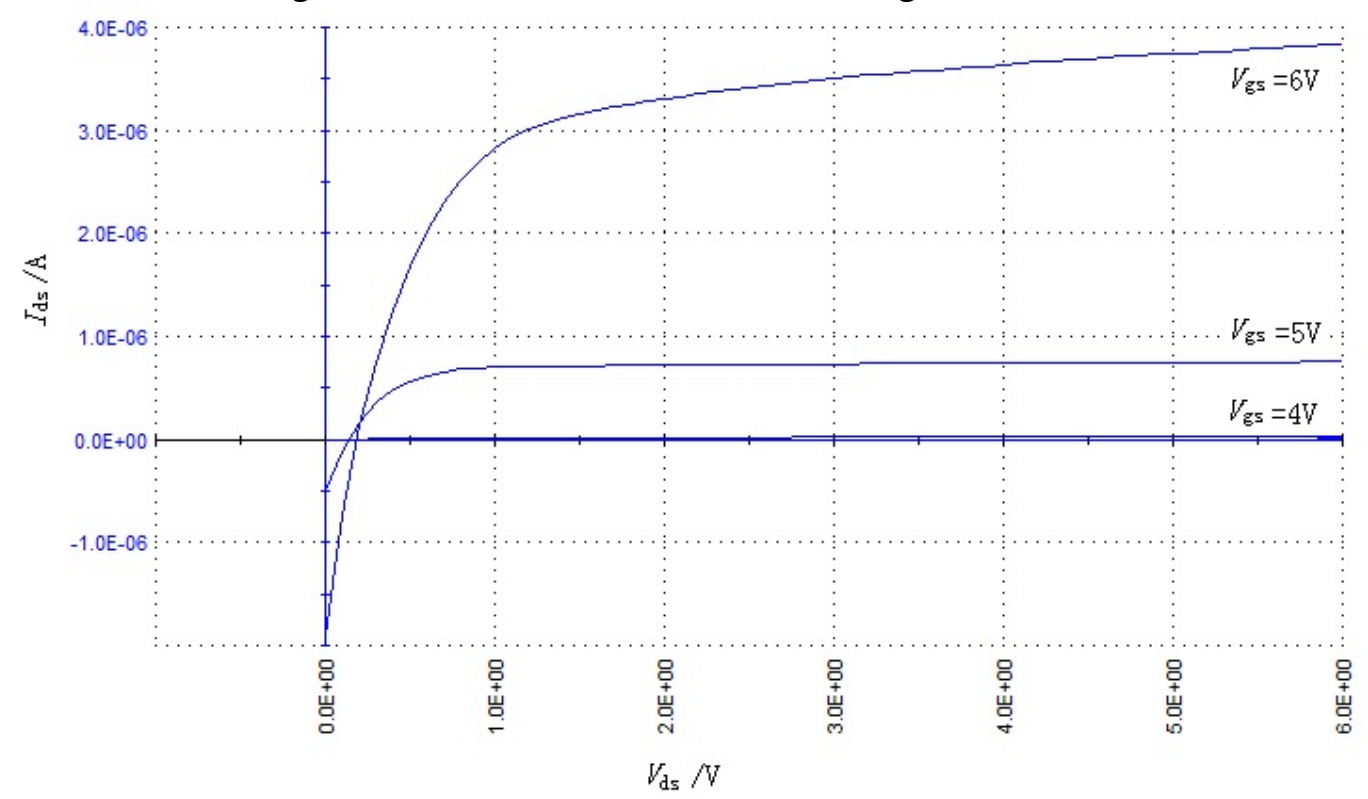

Fig. 4 Transmission characteristic curve 


\section{Conclusion}

A layer of a-IGZO thin film with a length and width of 50 um was deposited on high doped Ptype Si substrate by magnetron sputtering here. Some tests of the IGZO-TFT were carried out by using probe table and Keithley 4200 Semiconductor Parameter analyzer. The results show that the threshold voltage of the device is $4.5 \mathrm{~V}$, the sub-threshold swing is small, and the gate bias has a good regulation effect on the leakage current. The IGZO-TFT has good clamping characteristics and its electrical characteristics are also good.

\section{References}

[1] CH Lee, TH Kim, et al. Properties of IGZO Film Deposited by Ar/O2 Inductively Coupled Plasma Assisted DC Magnetron Sputtering [J]. Science of Advanced Materials, 2017, 9(7): 11871192.

[2] S Steudel, JLPJVD Steen, et al. Journal of the Society for Information Display [J], 2017, 25(4): 222-228.

[3] T Goto, F Imaizumi, et al. Improvement in the Negative Bias Illumination Stress Stability for Silicon-Ion Implanted Amorphous InGaZnO Thin-Film Transistors[J]. IEEE Electron Device Letters, 2017, 38(3): 345-348.

[4] SI Oh, G Choi, et al. Hydrogenated IGZO Thin-Film Transistors Using High-Pressure Hydrogen Annealing [J]. IEEE Transactions on Electron Devices, 2013, 60(8): 2537-2541.

[5] M Nag, A Chasin. New Polymer-Capped a-IGZO TFT with High Sensitivity to Visible Light for the Development of Integrated Touch Sensor Array [J]. Journal of the Society for Information Display, 2013, 21(3):129-136. 